\title{
LIBR: Roteamento Baseado em ID para Redes Sem Fio em Malha Lineares
}

\author{
Bruno B. Siqueira, Célio V. N. Albuquerque e Débora C. M. Saade
}

\begin{abstract}
Resumo- Este artigo propõe o LIBR, um protocolo de roteamento para Redes em Malha Sem Fio especializado em topologias lineares. Tal protocolo tem aplicação direta em redes de telemetria, automação e comunicação multimídia ao longo de linhas de transmissão de energia, gasodutos e vias. Para tanto, requisitos como simplicidade, escalabilidade, baixo tráfego de controle e aproveitamento das características da topologia linear são essenciais para o seu bom desempenho. Implementações do LIBR feitas no simulador NS-2 mostraram ganhos de vazão de 9\% ou mais quando comparado com outros protocolos de estado da arte.
\end{abstract}

Palavras-Chave-Redes Sem Fio em Malha, topologia linear, protocolo de roteamento, LIBR.

Abstract - This article proposes LIBR, a routing protocol for Wireless Mesh Networks specialized in linear topologies. Such protocol is applicable to telemetry or automation networks through power transmission lines, pipelines and roads. Features as simplicity, scalability, low overhead and use of linear topology specificities are part of the requirements for its good performance. Implementations of LIBR in NS-2 simulator showed that it has a gain of $9 \%$ or more of throughput when compared to state of the art protocols.

Keywords-Wireless Mesh Networks, linear topology, routing protocol, LIBR.

\section{INTRODUÇÃO}

Redes em malha sem fio (ou Wireless Mesh Networks $W M N s$ ), tecnologia chave da próxima geração de acesso sem fio, têm sido amplamente implantadas devido a sua flexibilidade e baixo custo, especialmente em locais onde a infraestrutura é inexistente e economicamente inviável. Redes em malha são redes sem fio autoconfiguráveis e com infraestrutura fixa, geralmente no padrão IEEE $802.11 \mathrm{em}$ modo ad-hoc, que interconectam um conjunto de nós estacionários capazes de rotear pacotes entre si através de múltiplos saltos.

Se por um lado redes em malha têm propriedades de boa cobertura e robustez, por outro sofrem de escalabilidade em termos de vazão, atraso e entrega de pacotes. As principais razões para isso passam pela baixa taxa de transmissão quando comparada a redes cabeadas, propriedade half-duplex dos rádios, interferência e dificuldade de se evitar colisões. Muitos trabalhos propõem soluções para essas questões usando antenas direcionais, adotando mecanismos de controle de potência e de taxa de transmissão, adotando múltiplos canais e percursos [2] ou mesmo implementando protocolos e métricas de roteamento eficientes [3].
O desempenho de uma rede em malha depende criticamente do seu diâmetro, onde diâmetro de rede é definido pelo menor caminho entre os nós mais distantes. Alguns estudos mostram que a vazão e o atraso fim-a-fim degradam rapidamente à medida que se aumenta o número de saltos [1]. Implantações de redes em malha com topologia linear, onde o diâmetro da rede é máximo dado um número de nós, demandam portanto uma atenção especial no tratamento do seu desempenho.

Redes em malha com topologia linear [8, 9] e grande diâmetro são aplicáveis à comunicação de telemetria, automação e dados multimídia ao longo de linhas de transmissão de energia elétrica, gasodutos, oleodutos, estradas, linhas de trem, etc. Um dos desafios das redes com essas características é implementar técnicas e protocolos que melhorem o desempenho da rede de forma escalável e com baixa sobrecarga de pacotes de controle.

O presente trabalho propõe o LIBR (Linear ID-Based Routing), um protocolo de roteamento para redes em malha sem fio especializado em topologia linear. Para tanto, requisitos como simplicidade, escalabilidade, baixo tráfego de controle e aproveitamento das características da topologia linear são essenciais para o seu bom desempenho.

Com o protocolo LIBR, os nós da rede têm conhecimento apenas da vizinhança local e dos gateways, que são os nós que conectam à Internet ou a outra rede privada com fio. Levando em conta que geralmente a maior parte do tráfego é destinado ou originado pelo gateway, o LIBR opera de maneira híbrida: um roteamento do tipo vetor distância avançado para comunicação com o gateway e um roteamento heurístico baseado na identificação do nó para comunicação entre demais nós. Para ambos os modos, o LIBR utiliza métrica ciente de qualidade de enlace, conforme classificação em [10].

Este artigo é organizado apresentando-se primeiramente trabalhos relacionados. Em seguida, explica-se com mais detalhes o funcionamento do protocolo LIBR em seus diferentes modos. Na Seção IV, são mostrados resultados de simulações que comparam o LIBR a outros protocolos e, na conclusão, são feitas as considerações finais e indicados trabalhos futuros.

\section{TRABALHOS RELACIONADOS}

Redes em malha se fio diferem significativamente das redes convencionais, especialmente no que diz respeito a interconectividade dos nós. Um dos primeiros protocolos de roteamento propostos para redes sem fio ad-hoc foi o DSDV 
(Destination-Sequenced Distance-Vector) [4]. O DSDV é um protocolo do tipo vetor distância em que os nós vizinhos trocam periodicamente suas tabelas de roteamento com a contagem de saltos até cada um dos nós da rede. A tabela de roteamento é atualizada preferencialmente com as rotas com número de sequência mais recente e depois com aquelas de menor contagem de saltos.

O protocolo OLSR (Optimized Link State Routing) [5] é um protocolo de estado de enlace onde os nós conhecem toda a topologia de enlaces e difundem essa informação pela rede. $\mathrm{O}$ ponto forte do OLSR é o controle da inundação através dos nós MPR (multipoint relays), que formam o menor conjunto de vizinhos a um salto que alcançam todos os vizinhos a dois saltos.

Numa proposta diferente das demais, o AODV (Ad hoc OnDemand Distance Vector) [6] se apresenta como um protocolo reativo onde as rotas são formadas quando há demanda de tráfego. Com mensagens de solicitação de rota e de resposta, o protocolo forma caminhos por vetor distância apenas para os destinos com interesse de tráfego.

Em termos de métricas de roteamento, a primeira proposta foi a contagem de saltos, a qual considera o melhor caminho aquele com a menor contagem de saltos. Essa métrica, todavia, não leva em conta a qualidade dos enlaces sem fio e, na prática, não possui um bom desempenho [10].

Dentre as métricas de roteamento cientes de qualidade de enlace, a métrica ETX (Expected Transmission Count) [7] estima o número esperado de transmissões para se enviar com sucesso um pacote entre dois nós. A estimativa é calculada a partir da taxa de entrega de pacotes num enlace, em seus dois sentidos.

No que diz respeito a redes em malha sem fio lineares, já foi mostrado que tais redes atendem bem à necessidade de uma infraestrutura de comunicação nos ambientes de linhas de transmissão de energia [8]. Alguns trabalhos inclusive apresentam algoritmos para um planejamento ótimo de tais redes [9].

\section{PROTOCOLO LIBR}

O protocolo de roteamento LIBR, proposto neste trabalho, foi projetado levando em conta que a maior parte do tráfego em uma rede em malha sem fio tem o gateway como origem ou destino. Por essa razão, o protocolo escolhe o melhor caminho até o gateway usando um roteamento vetor distância. Comparado a outros protocolos do tipo vetor distância, como DSDV e AODV, essa seletividade reduz consideravelmente o volume de tráfego de controle, sendo trocadas apenas informações para formação de rota até o gateway. Para fluxos entre demais nós, o protocolo usa uma heurística baseada na identificação dos nós (ID) aproveitando das características da topologia linear. Para ambos os modos, o protocolo usa a métrica ETX ciente de qualidade de enlace.

\section{A. Roteamento baseado em ID}

Esse método de roteamento é uma heurística que compara o ID do destino do pacote com o ID dos vizinhos para tomar decisões de encaminhamento. Para isso, o roteador precisa ter conhecimento apenas dos vizinhos a um salto, dispensando o conhecimento de toda a rede. Redes de topologia linear geralmente são planejadas e acompanham algum outro sistema ou via, de maneira que torres, postes ou marcações são naturalmente identificados sequencialmente. Tomando proveito dessa natureza sequencial, os roteadores simplesmente encaminham o pacote ao vizinho com ID mais próximo do ID de destino.

Quando o destino do pacote é um dos vizinhos, o roteador compara a qualidade dos enlaces da vizinhança local para decidir se o encaminhamento do pacote será feito de forma direta ou indireta por intermédio de outro vizinho. $\mathrm{O}$ encaminhamento indireto também é usado no roteamento baseado em ID. A Figura 1 ilustra um exemplo em que o nó 2 quer enviar um pacote ao nó $\mathbf{5}$. O nó $\mathbf{2}$ não conhece o nó $\mathbf{5}$ mas sabe por cálculo da diferença que o vizinho 4 é o mais próximo do destino. Tendo ciência dos custos dos enlaces da vizinhança, o nó 2 decide encaminhar o pacote ao nó 3 , pois o custo do caminho com dois saltos $(1,0+1,5)$ é menor que o custo do enlace direto ao nó 4 (3,0). O nó $\mathbf{3}$, por sua vez, fará nova decisão de rota e não necessariamente encaminhará o pacote ao vizinho 4.

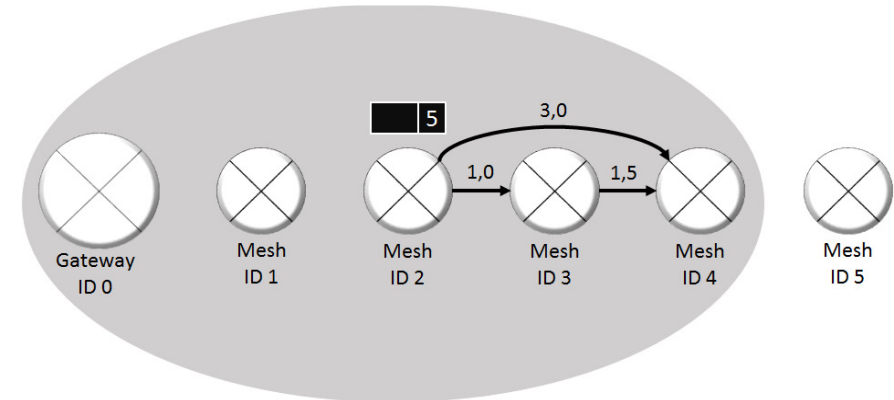

Fig. 1. Exemplo de roteamento baseado em ID (a área cinza representa o alcance do nó 2 ..

A atribuição de ID aos nós deve ser feita de forma que não viole a ordem sequencial para não causar loop de roteamento. Diferentes critérios de projeto para atribuição de ID podem ser adotados, como número de saltos a partir do gateway, numeração de linha ou sistema que acompanha a rede ou distância física quantizada a partir do gateway. Seja qual for o critério adotado, é recomendável reservar lacunas na sequência prevendo mudanças por ampliação ou manutenção da rede.

A adoção desse tipo de roteamento exige um mecanismo de mapeamento de endereço IP para ID, uma vez que os pacotes de dados não carregam a informação de ID. No intuito de se evitar sobrecarga de pacotes de controle tanto pela difusão na rede como pela consulta sob demanda dessa informação, é proposto um mapeamento direto de parte dos bits do endereço IP para o ID. O mecanismo é adequado para redes lineares planejadas que sofram poucas mudanças. Como exemplo, dada a rede IP 192.168.0.0 /24, pode ser usado o último octeto do endereço para formação de um ID de 8 bits.

\section{B. Roteamento vetor distância}

No protocolo de roteamento vetor distância padrão, vizinhos trocam entre si mensagens periódicas com suas tabelas de roteamento contendo a contagem de saltos para cada destino da rede. Com base nessas informações, o nó encaminha um pacote de dados àquele vizinho com a menor distância até o destino.

No protocolo LIBR proposto, algumas mudanças são feitas sobre o roteamento vetor distância padrão de maneira melhorar o seu desempenho para redes sem fio em malha com topologia linear. A primeira delas é habilitar o roteamento vetor distância apenas às rotas de destino aos gateways da rede, uma vez que a maior parte do tráfego é destinado a eles. Isso já reduz 
consideravelmente o tamanho das mensagens de controle, diminuindo a probabilidade de colisão bem como a sobrecarga do controle.

Como apenas os gateways divulgam suas rotas, eles não aprendem rotas para os demais nós da rede. Com o objetivo de se contornar o problema de rotas assimétricas e se manter o requisito de rotas ótimas para fluxos indo e vindo do gateway, é adotado o roteamento por caminho reverso. Para todo pacote destinado ao gateway, cada nó da rede salva em sua tabela de roteamento o endereço de origem e o salto anterior do pacote. Dessa forma, quando a resposta voltar do gateway, o nó saberá a que vizinho encaminhá-la usando o mesmo caminho de ida.

Os problemas de contagem a infinito e loop de roteamento são evitados por meio de duas técnicas: divulgação do número de sequência e divulgação do nó por onde foi aprendida a rota. Toda vez que um gateway divulga sua rota para propagação pela rede, ele estampa a mensagem com um número de sequência. Uma vez que a rede atinja um estado estável, é demonstrado por teoria dos grafos em [4] que se forma uma árvore livre de loop entre a raiz representada pelo gateway e cada um dos demais nós. Essa condição será atendida se:

1. Cada nova rota contiver o número de sequência mais recente;

2. Dadas rotas com o mesmo número de sequência, for escolhida aquela com o menor custo.

Todavia, a perda de uma mensagem de controle com um novo número de sequência pode quebrar a primeira condição citada acima. Por essa razão e como melhoria à técnica utilizada pelo protocolo DSDV, junto à rota divulgada é informado também por qual nó a rota foi aprendida. Caso um nó receba de seu vizinho uma rota que foi aprendida por ele próprio, ele descarta essa rota. Essa técnica é análoga às técnicas Split Horizon e Poison Reverse usadas pelo protocolo RIP (Routing Information Protocol), porém como numa rede sem fio os nós compartilham um mesmo domínio de broadcast, não faz sentido omitir uma rota ou divulgá-la com métrica infinita.

Uma terceira mudança do protocolo vetor distância padrão é o uso de métrica ciente da qualidade de enlace no lugar da métrica de contagem de saltos. Como comentado anteriormente, a métrica ETX calcula o número esperado de transmissões a partir das probabilidades de entrega de um enlace. O ETX é calculado por:

$$
E T X=\frac{1}{d_{f} \times d_{r}}
$$

A taxa de entrega para frente, $d_{f}$, é medida pela probabilidade de um pacote de dados ser recebido com sucesso pelo nó receptor, e a taxa de entrega reversa, $d_{r}$, é medida pela probabilidade de um pacote ACK ser recebido com sucesso pelo nó transmissor. Para efeitos do protocolo, apenas os pacotes de controle são usados para cálculo das probabilidades.

O uso da métrica ETX aumenta consideravelmente a vazão média da rede pois define o caminho com o menor número médio de transmissões. Para o devido cálculo da métrica, se faz necessária a troca de informações entre vizinhos por mensagens periódicas de controle, apresentadas na seção seguinte.

\section{Mensagens de controle}

Mensagens de controle são trocadas periodicamente entre vizinhos em modo broadcast, sendo sugerido um intervalo de 15 segundos, conforme utilizado pelo protocolo DSDV. Além dos pacotes periódicos, pacotes intempestivos podem ser enviados informando imediatamente a queda de algum nó, por meio de métrica infinita, ou informando o surgimento de um novo gateway na rede. A queda de um nó vizinho é percebida após determinado tempo sem receber mensagens de controle e a queda de um gateway é percebida após determinado tempo sem atualizações com novos números de sequência.

Os pacotes de controle divulgam informações de vizinhança e de gateways através dos campos mostrados na Figura 2.

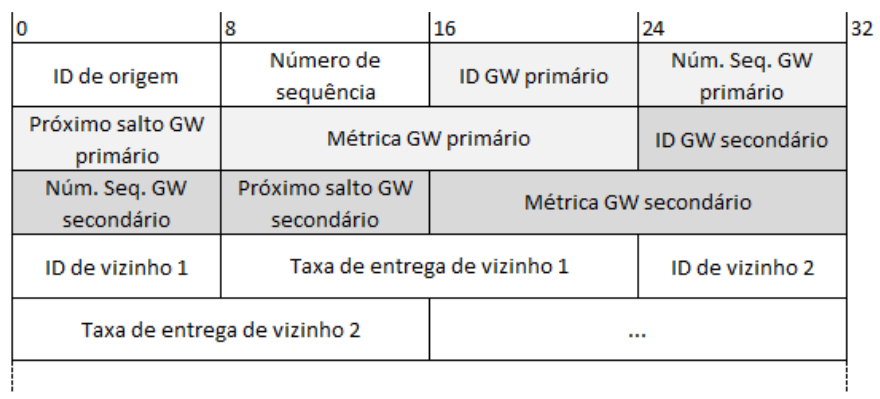

Fig. 2. Campos do pacote de controle do LIBR

O tamanho do pacote LIBR é de 12 bytes fixos mais 3 bytes por vizinho, limitado a 10 vizinhos, de maneira que seu tamanho máximo é de 42 bytes. Os dois primeiros campos, ID de origem e número de sequência, são usados para calcular a taxa de recebimento de pacotes de um determinado vizinho.

Os oito campos seguintes são referentes às informações dos gateways. A métrica até o gateway é calculada pela soma da métrica divulgada mais a métrica do enlace com o vizinho que originou o pacote. Mesmo que a rede tenha mais de dois gateways, são divulgados apenas um gateway primário e um secundário, aqueles com as melhores métricas. Esse método visa superar uma das limitações dos protocolos vetor distância referente a convergência da rede, fazendo com que os nós comutem rotas rapidamente de um gateway primário para um gateway secundário em caso de queda do primeiro.

TABELA I. MODOS DE FUNCIONAMENTO DO LIBR

\begin{tabular}{|l|l|l|}
\hline \multicolumn{1}{|c|}{ ORIGEM } & \multicolumn{1}{|c|}{ DESTINO } & \multicolumn{1}{c|}{ ROTEAMENTO } \\
\hline Nó mesh & Vizinho a um salto & $\begin{array}{l}\text { Menor ETX pela } \\
\text { vizinhança local }\end{array}$ \\
\hline Nó mesh & $\begin{array}{l}\text { Nó mesh fora da } \\
\text { vizinhança }\end{array}$ & \begin{tabular}{l} 
Baseado em ID \\
\hline Nó mesh
\end{tabular} \\
Gateway & $\begin{array}{l}\text { Vetor distância } \\
\text { com métrica ETX }\end{array}$ \\
\hline Gateway & Nó mesh & Caminho reverso \\
\hline
\end{tabular}

Os demais campos do pacote informam o ID e a taxa de entrega dos vizinhos do nó remoto que originou o pacote. Com a taxa de entrega divulgada e a taxa de recebimento calculada, é possível computar a métrica ETX de um nó a todos seus vizinhos de um salto, assim como a métrica ETX entre dois 
vizinhos diferentes, permitindo o cálculo da melhor rota dentro da vizinhança. A Tabela I resume os diferentes modos de funcionamento do protocolo LIBR.

\section{AVALIAÇÃO DE DESEMPENHO}

O protocolo LIBR foi implementado em C++ no simulador NS-2 e comparado com os protocolos DSDV, AODV, OLSR com métrica ETX. Para fins de avaliação da contribuição, o LIBR foi simulado no modo de roteamento baseado em ID.

\section{A. Simulações}

As simulações no NS-2 foram executadas com 40 nós em topologia linear, com um diâmetro de rede próximo a uma rede em malha real descrita em [8], com nós igualmente espaçados a 70 metros de maneira a alcançar vizinhos de até três saltos. $\mathrm{O}$ modelo de propagação utilizado foi o Two Ray Ground, com taxa de transmissão na camada física de $11 \mathrm{Mbps}$ para pacotes de dados e 2 Mbps para pacotes de controle. Foram estabelecidos dez fluxos TCP do primeiro ao último nó, de maneira a simular o download de dados por aplicações TCP e maximizar os impactos do diâmetro da rede sobre seu desempenho.

As simulações foram executadas durante 300 segundos, sendo os primeiros 100 segundos reservados para convergência da rede e os últimos 200 segundos com fluxos de dados. Foram feitas medições de vazão útil média, latência média e sobrecarga de bytes de controle. Para cada protocolo, foram feitas 10 simulações com 10 sementes aleatórias diferentes. Os gráficos exibem as médias das simulações executadas.

\section{B. Resultados}

Abaixo são mostrados os resultados em gráficos de barra das medidas de vazão útil média, atraso médio e sobrecarga de bytes de controle, com o intervalo de confiança de $95 \%$, comparando o LIBR com os protocolos DSDV, AODV e OLSR com métrica ETX.

A Figura 3 mostra a vazão útil média para os quatro protocolos comparados, calculada pelo volume de bytes de segmentos TCP não repetidos recebidos na camada de aplicação dividido pelo tempo total de transmissão de dados. $\mathrm{O}$ LIBR mostra-se amplamente superior ao AODV e ao DSDV, e 9,1\% melhor que OLSR-ETX. A Figura 4 compara a latência média, calculada pelo tempo médio de envio de dados fim-afim. Nessa comparação, observa-se que o LIBR apresentou um resultado próximo ao do OSLR-ETX, com um atraso médio de 116 milissegundos, estando apenas 8 milissegundos acima do OLSR-ETX. Essa pequena diferença se deve a retransmissões e a maiores tempos de acesso ao meio causados pelo maior volume de dados transmitidos. Por fim, na Figura 5, fica evidenciado que o LIBR tem a menor sobrecarga de controle ou overhead, medida pela razão de bytes de controle transmitidos pelo total de bytes transmitidos.

Apesar do LIBR não ter conhecimento da topologia de toda a rede, sua heurística de roteamento baseado em ID apresentou bom desempenho quando comparada a outros protocolos de roteamento conhecidos no meio acadêmico e utilizados em redes em malha reais. Uma das razões desse bom desempenho se deve ao uso da métrica ETX ciente de qualidade de enlace, que auxilia na escolha do melhor caminho dentro da vizinhança. Uma outra explicação para a boa vazão se deve à baixa sobrecarga de controle, que deixa o meio mais livre para fluxos de dados.

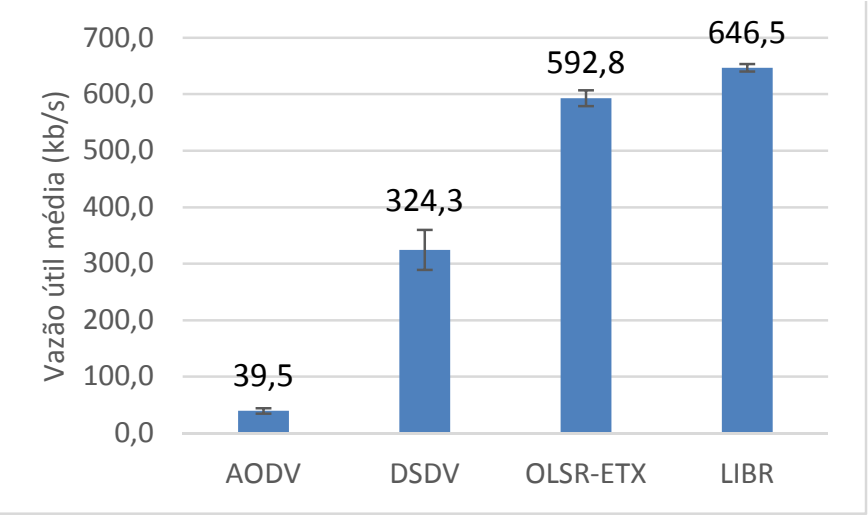

Fig. 3. Comparação de vazão útil média

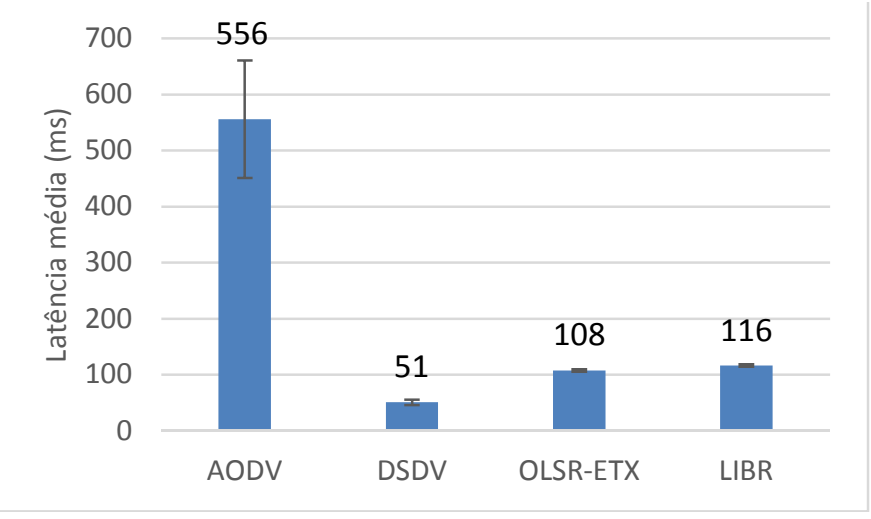

Fig. 4. Comparação de latência média

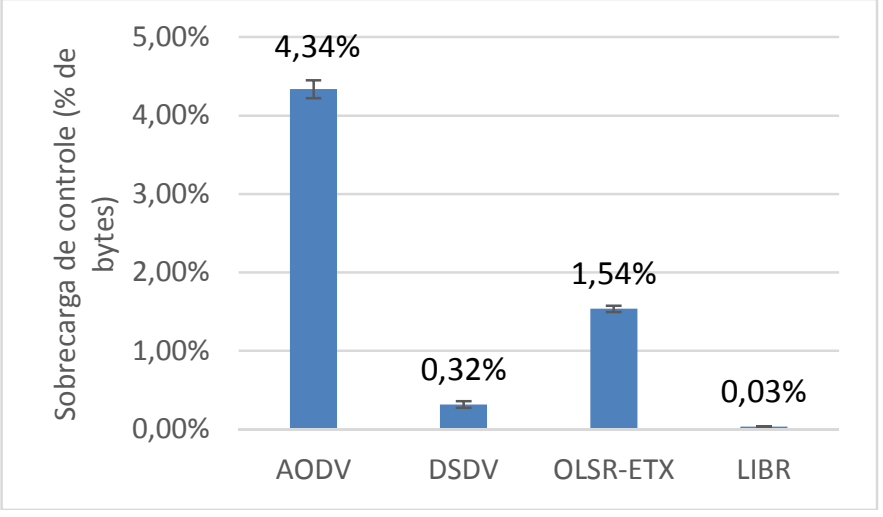

Fig. 5. Comparação de sobrecarga de bytes de controle

\section{CONCLUSÕES}

Nesse artigo foi apresentado o protocolo de roteamento LIBR para redes sem fio em malha com topologia linear, as quais apresentam maiores desafios de desempenho. Com um modo de funcionamento híbrido de vetor distância e baseado em ID, o protocolo atende aos requisitos exigidos de simplicidade, escalabilidade e baixo tráfego de controle

Nas simulações, o LIBR alcançou os melhores resultados quando comparado aos protocolos conhecidos OLSR-ETX, AODV e DSDV, especialmente no que tange a vazão. Um dos 
principais motivos desse bom desempenho se deve à baixa sobrecarga de controle. O modo de roteamento baseado em ID abre oportunidades para novos desenvolvimentos em rede em malha sem fio de grande diâmetro.

Como trabalhos futuros, pretende-se avaliar o impacto do LIBR sobre o consumo de energia da rede e implementá-lo numa rede em malha sem fio real que utiliza roteadores com o OpenWRT.

\section{AGRADECIMENTOS}

Agradecemos a todos aqueles que contribuíram com ideias do LIBR, em especial ao Diego Passos por colaborar não só com sugestões e melhorias como também com informações úteis sobre o NS-2.

\section{REFERÊNCIAS}

[1] H. Skalli, S. Ghosh, S. K. Das, L. Lenzini e M. Conti, Channel assignment strategies for multiradio wireless mesh networks: issues and solutions, IEEE Communications Magazine, 2007.
[2] Tam, W. and Tseng, Y. (2007) "Joint Multi-Channel Link Layer and Multi-Path Routing Design for Wireless Mesh Networks", In: INFOCOM.

[3] D. Passos, e C. Albuquerque, "A Joint Approach to Routing Metrics and Rate Adaptation in Wireless Mesh Networks", IEEE/ACM Transactions on Networking, v. 20, pp. 999-1009, Agosto 2012.

[4] C. Perkins e P. Bhagwat, "Highly Dynamic Destination-Sequenced Distance-Vector Routing (DSDV) for Mobile Computers", SIGCOMM, pp. 234-244, 1994.

[5] T. Clausen, P. Jacquet, C. Adjih, A. Laouiti, P. Minet, P. Muhlethaler, A. Qayyum e L. Viennot, "Optimized link state routing protocol (OLSR)", Inria, 2003.

[6] C. Perkins e E. Royer, "Ad-hoc on-demand distance vector routing." Mobile Computing Systems and Applications (WMCSA), 1999.

[7] D. De Couto, "High-Throughput Routing for Multi-Hop Wireless Networks", tese PhD, Massachusetts Institute of Technology, 2004.

[8] L. Gerk, D. Passos, D. Saade e C. Albuquerque, "Infraestrutura de comunicação em malha sem fio para supervisão e controle de sistemas de transmissão de energia", Espaço Energia, pp 9-18, Abril 2009.

[9] F. Souza e C. Albuquerque, "Linear wireless mesh network planning." International Information and Telecommunication Technologies Symposium (I2TS), 2010.

[10] M. Campista, P. Esposito, I. Moraes, L. Costa, O. Duarte, D. Passos, C. Albuquerque, D. Saade e M. Rubistein, "Routing metrics and protocols for wireless mesh networks. Network", IEEE Network, v. 22, pp. 6-12, Fevereiro 2008. 